\title{
Designing Robustness to Temperature in a Feedforward Loop Circuit
}

\author{
Shaunak Sen, Jongmin Kim, Richard M. Murray
}

\begin{abstract}
Incoherent feedforward loops' represent important biomolecular circuit elements capable of a rich set of dynamic behavior including adaptation and pulsed responses. Temperature can modulate some of these properties through its effect on the underlying reaction rate parameters. It is generally unclear how to design a circuit where these properties are robust to variations in temperature. Here, we address this issue using a combination of tools from control and dynamical systems theory as well as preliminary experimental measurements towards such a design. Using a structured uncertainty representation, we analyze a standard incoherent feedforward loop circuit, noting mechanisms that intrinsically confer temperature robustness to some of its properties. Further, we study design variants that can enhance this robustness to temperature, including different negative feedback configurations as well as conditions for perfect temperature compensation. Finally, we find that the response of an incoherent feedforward loop circuit in cells can change with temperature. These results present groundwork for the design of a temperature-robust incoherent feedforward loop circuit.
\end{abstract}

\section{INTRODUCTION}

Living cells are subject to a wide variety of environmental changes, for example, due to seasonal changes in variables like temperature and humidity. Biomolecular circuits regulating their behavior are likely to possess the property that ensures robust functionality in face of such environmental changes [1]. A similar property can be desirable for engineered biomolecular circuit designs, whereby they also function robustly in a range of environments, as well as for allowing circuit modules designed to function in slightly different environments to be reliably interconnected. In particular, temperature is an important environmental variable that can modulate the functional output of biomolecular circuit designs as a direct consequence of its effect on the underlying reaction rate parameters. Indeed, for biomolecular circuit designs, temperature has been identified as a key element of the environmental context against which functional robustness needs to be assessed [2].

Mathematical frameworks to investigate such robustness issues include classical control theoretic notions such as robust tracking as well as generalization of these classical ideas to account for the rich, complex behavior of biomolecular systems. Examples are investigations of perturbation classes

Research supported in part by the Gordon and Betty Moore Foundation and the NSF Molecular Programming Project.

S. Sen is with the Department of Electrical Engineering, Indian Institute of Technology Delhi, New Delhi 110016, INDIA. E-mail: shaunak. sendee.iitd.ac.in

J. Kim is with the Wyss Institute, Harvard University, Boston, MA 02115 , USA. E-mail: jongmin.kim@wyss.harvard.edu

R. M. Murray is with the Divisions of Engineering and Applied Science and Biology and Biological Engineering, California Institute of Technology, Pasadena, CA 91125, USA. E-mail: murray@cds.caltech. edu

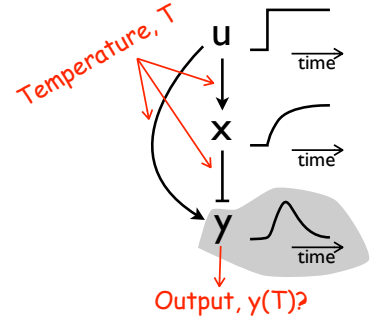

Fig. 1. Temperature can affect the output of an incoherent feedforward loop through its effect on the underlying reactions. A standard representation of such a circuit is sketched. In response to an input step, there can be a pulsed output. Red arrows indicate the propagation of temperature dependence. The use of the term 'feedforward' to describe such a circuit may have originated to describe the direct link between input $u$ and output $y$.

under which the transient response is invariant [3], [4] and of structural attributes of the biomolecular reaction network guaranteeing robustness of the steady-state response [5], [6]. Insofar as temperature robustness in biomolecular circuits is concerned, the most studied systems have been limit cycle oscillators, both natural [7] and synthetic [8]. In these systems, temperature robustness manifests in the oscillation period, which is approximately constant over a range of temperatures. One way to characterize robustness has been to define a temperature coefficient $Q_{10}(T)$ of a temperaturedependent rate/property $k(T)$, as the ratio $k(T+10) / k(T)$. A $Q_{10}=2$ implies that $k$ doubles for a $10^{\circ} \mathrm{C}$ increase in temperature. In contrast, a $Q_{10}=1$ means that $k$ does not change with temperature. It is often found that $Q_{10}$ of the oscillation period is close to 1 , indicating temperature robustness, while the typical $Q_{10}$ values for biomolecular reaction rates range from 2-3 [9]. A recent example where temperature robustness has also been studied is bacterial chemotaxis [10]. In these examples, temperature-dependent elementary reaction rates cancel the effects of each other exactly such that the functional output is temperature-robust. Temperature compensation has also been recently reported for a designed biomolecular oscillator [11]. These studies provide important early work towards the design of temperature-robust biomolecular circuit behavior.

Feedforward loop circuits represent an interesting class of biomolecular circuits with at least three striking features [12]. First is their widespread occurrence in different biomolecular contexts, underscored by their overrepresentation in transcriptional networks [13]. Second is their systems level properties, for example, the incoherent feedforward loop (Fig. 1) can exhibit both perfect adaptation [14], [15] and fold-change detection [16], [17]. Third is the dynamical outputs that can 
be achieved, for example, the incoherent feedforward loop can generate pulse dynamics, whose quantitative properties such as the pulse height, adaptation value, rise time, and decay time can be of functional importance in cells. How can temperature affect these properties? Can modifications to the circuit design enhance the temperature robustness? Indeed, it is generally unclear how to design an incoherent feedforward loop circuit where properties are temperature-robust.

Here, we ask the question of how to design a temperaturerobust incoherent feedforward loop circuit. To address this issue, we use tools from control and dynamical systems theory. We investigate a standard incoherent feedforward loop circuit model using a structured uncertainty representation and note inherent features that promote temperature robustness. Next, we investigate design variants aimed at enhancing this robustness including the use of negative transcriptional feedback and conditions for perfect temperature compensation. Finally, as a step towards a robust design, we present preliminary experimental data illustrating how the dynamics of a circuit realization can change with temperature. These results should aid the design of a temperature-robust incoherent feedforward loop circuit.

\section{RELEVANT THEORETICAL TOOLS}

In order to understand how temperature affects functional output in biomolecular circuits and how this effect may be compensated for, we first adapt relevant tools from control in this context. Consider a mathematical representation of the dynamics of a biomolecular circuit obtained using standard mass-action-based ordinary differential equations,

$$
\frac{d x}{d t}=f(x, \mu)
$$

Here, $x$ is a vector of concentration variables and $\mu$ is a vector of reaction rate parameters. Because of the temperature dependence of reaction rate parameters $\mu=\mu(T)$, for example, as characterized by a temperature coefficient $Q_{10}^{\mu}$, functional properties such as steady-state levels or transient features can also be temperature dependent.

Uncertainty Representations. In fact, using this characterization, effect of temperature in Eqn. (1) can be analyzed like a structured uncertainty in the reaction rate parameters. For example, the range of $Q_{10}$ 's of the outputs under consideration can be computed for a given set of $Q_{10}$ 's of the reaction rate parameters. Alternatively, these parametric uncertainties map to a range of values for each output under consideration, which can be directly considered. In particular, the temperature dependence of the steady-state values $x_{0}$ can be obtained directly from the algebraic equation $f\left(x_{0}, \mu\right)=$ 0 . Other potentially useful uncertainty representations can also be obtained through linearization of Eqn. (1) around a desired operating point $x=x_{0}$.

For example, consider a simple model of protein production-degradation,

$$
\frac{d x}{d t}=\beta-\gamma x
$$

where $x$ is the concentration of a protein, produced at a constant rate $\beta$, and degraded as a first-order process with rate constant $\gamma$. At steady state, $x_{s s}=\beta / \gamma$. The temperature dependence in this model can be analyzed by considering the parametric uncertainty in $\beta$ and $\gamma$ due to temperature.

Internal Model Principle. One way to reduce the effect of such uncertainty is through the use of a control strategy. Indeed, different control strategies may minimize the effect of the variation of temperature on the circuit performance to different degrees. In order that perfect temperature compensation be achieved, a natural question arises with regard to the properties that such a controller must possess. In fact, this question bears resemblance to the Internal Model Principle [18], [19], [20], which requires that the system possess a model of the external signal that it needs to adapt against. For example, perfect adaptation to step inputs can be implemented through the use of an integrator block in the loop transfer function. If the input was a ramp, the presence of a single integrator block would not suffice for perfect adaptation and a double integrator would be required.

A similar guideline is likely to be relevant for perfect temperature compensation, where it is desired for an output to be invariant to temperature even though underlying circuit parameters change with temperature. Consider an augmentation to the simple model above with a control input $u$ that is assumed to be able to directly control the rate of change of $x$,

$$
\frac{d x}{d t}=\beta-\gamma x+u
$$

We consider the steady state $x_{s s}$ as the output of this system. Suppose both parameters change with temperature, $\beta=\beta(T), \gamma=\gamma(T)$ Perfect temperature compensation can be achieved if these temperature dependencies are known. A control input that achieves this is $u=-(\beta(T)-$ $\left.\beta\left(T_{0}\right)\right)-\left(\gamma(T)-\gamma\left(T_{0}\right)\right) x$, where $T_{0}$ is a reference temperature. Therefore, knowledge of temperature dependence and possibility of such cancellation enables perfect temperature compensation.

An adaption of these tools in the context of temperature robustness illustrates ways to represent temperature dependence and the ideal conditions for a system response to be temperature independent. In the following, we focus on a standard incoherent feedforward loop circuit model.

\section{INHERENT TEMPERATURE ROBUSTNESS IN AN INCOHERENT FEEDFORWARD LOOP MODEL}

To characterize possible temperature dependence of the properties of an incoherent feedforward loop, we consider a mathematical model of it (adapted from [17]),

$$
\begin{aligned}
& \frac{d x}{d t}=\alpha_{x} u-\gamma x, \\
& \frac{d y}{d t}=\alpha_{y} u \frac{K_{x}}{x}-\gamma y .
\end{aligned}
$$

Here, $u$ is the input, $x$ is the intermediate variable, and $y$ is the output. The parameters $\alpha_{x}, \alpha_{y}, \gamma$, and $K_{x}$ represent the production rate of $x$, of $y$, their dilution rates, and the binding 

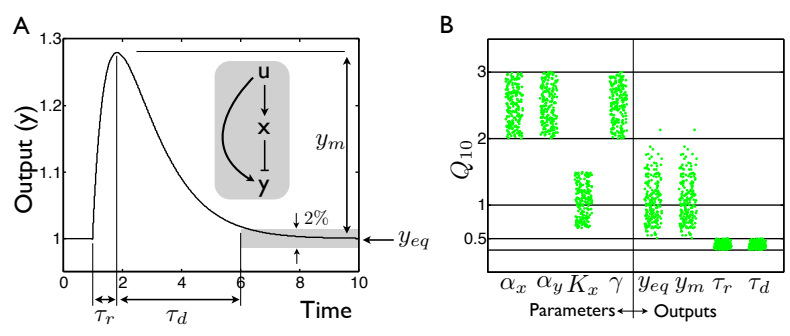

Fig. 2. Propagation of temperature dependence in a model of an incoherent feedforward loop circuit. A. Black line is a trajectory computed from the model. Different properties are graphically illustrated on the trajectory. Inset shows a schematic illustration of the circuit. B. Green dots illustrate the range of temperature coefficient's $\left(Q_{10}\right.$ 's) of the indicated reaction rate parameters and outputs. Horizontal spread is due to an arbitrary random number added for illustration purposes. Solid black lines indicate key $Q_{10}$ values.

constant of $x$ to the promoter of $y$, respectively. The origin of the term $K_{x} / x$ is as an approximation to a repression function like $K_{x} /\left(K_{x}+x\right)$. We assume that the default values of the parameters are $\alpha_{x}=\alpha_{y}=\gamma=K_{x}=u=1$. Further, we assume that a step change in the input leads to a change in the value of $u$ from 1 to 2 . This model exhibits the adaptation property (Fig. 2): In response to a step change in input $u$, both $x$ and $y$ first increase; As $x$ increases further, it represses $y$ and $y$ relaxes to its pre-step value. Therefore, the $y$ waveform exhibits a pulse in response to a step change in input $u$ (Fig. 2). This pulse shape can be further characterized by the following four properties: adaptation level $\left(y_{e q}\right)$, pulse amplitude $\left(y_{m}\right)$, rise time $\left(\tau_{r}\right)$, and decay time $\left(\tau_{d}\right)$. These properties depend on temperature dependent reaction rate parameters, and so can themselves be temperature dependent. The above model can be used to investigate the possible temperature dependencies.

We note that the adaptation property is solely dependent on the mechanism and is robust to changes in parameters. As such, it is also robust to parametric variations owing their origin to a temperature effect. In contrast, properties such as adaptation level, pulse amplitude, rise time, and decay time can depend on temperature through their dependence on circuit parameters. To investigate this dependence in the model, we make the following assumptions. First, we assume that the parameters take the default values specified above. Second, we assume that their temperature coefficient $Q_{10}$ is in the range $2-3$. Finally, based on this assumed range of $Q_{10}$ 's of the parameters, we calculate the range of temperature coefficient $Q_{10}$ of each of the properties.

As an illustration of this approach, consider the equilibrium value of $y_{e q}=K_{x} \alpha_{y} / \alpha_{x}$ obtained in the above model. In particular, let us consider the ratio $r=\alpha_{y} / \alpha_{x}$. Assuming that the $Q_{10}$ 's of $\alpha_{x}$ and $\alpha_{y}$ are in the range $2-3$, the $Q_{10}$ of $r$ has a minimum value of $2 / 3 \approx 0.66$ and a maximum value of $3 / 2=1.5$. As the DNA binding constant $K_{x}$ arises as a ratio of the off and on rates for the binding of $x$ to promoter of gene $y$, we assume that its $Q_{10}$ is also in the range $0.66-1.5$ (assuming these off/on rates also have $Q_{10}$ in range 2-3). Therefore, we estimate the $Q_{10}$ of $y_{e q}$ to be in the range $0.44-2.25$. In fact, this illustration captures a temperature compensation mechanism already inherent in this model. The temperature coefficient of the equilibrium level is different from those of the parameters because the parameters combine as a ratio of terms. Whenever two terms with similar temperature dependencies combine in a ratio, the effective temperature dependence of the ratio can be attenuated. Indeed, in this case, the production rates $\alpha_{x}$ and $\alpha_{y}$ are expected to have similar temperature dependencies, even if they do not strictly fall in the range of $Q_{10}$ 's assumed above. Therefore, this temperature compensation is expected to persist in a general setting as well and is an instance of the cancellation mentioned in the previous section.

Next, we apply this approach to other properties. As obtaining their analytical expressions is not as straightforward as that for the equilibrium value, we use numerical simulations. For this, we first choose $Q_{10}$ of each of the parameter as a different random number in the range 2-3. Using this selection, we compute the $Q_{10}$ for each of the above-mentioned properties: Pulse amplitude is computed as the maximum of the resulting waveform away from the equilibrium value. The rise time is computed as the time taken to reach the maximum pulse amplitude from when the pulse is applied. Similarly, the decay time is computed as the time it takes to go from when the pulse maximum is reached to when the response first returns to within $2 \%$ of its equilibrium value. The results of this computation for $N=200$ different random choices of $Q_{10}$ sets are shown in Fig. 2. As expected, we find that the $Q_{10}$ of the equilibrium value is in the range $0.44-2.25$. Similarly, the pulse amplitude is also in this range, indicating a similar compensation effect to a change in temperature. In contrast, the $Q_{10}$ 's of the rise time and decay time are in the range $0.33-0.5$, consistent with timescale being determined by the reciprocal of $\gamma$, a parameter with $Q_{10}$ in the range 2-3. Therefore, this indicates that other than a conversion of a $100 \%-200 \%$ increase in parameters to a $50 \%-66 \%$ reduction in the property, there is no other temperature robustness effect for the timescale-related parameters.

In order to gain insight into the temperature dependence of these properties, we considered a linearization of the Eqns. (4)-(5) about the operating point $u=u_{0}, x=x_{e q}=$ $\alpha_{x} u_{0} / \gamma$, and $y=y_{e q}$,

$$
\left[\begin{array}{c}
\delta \dot{x} \\
\delta \dot{y}
\end{array}\right]=\left[\begin{array}{cc}
-\gamma & 0 \\
-\frac{\alpha_{y} u_{0} K_{x}}{x_{e q}^{2}} & -\gamma
\end{array}\right]\left[\begin{array}{l}
\delta x \\
\delta y
\end{array}\right]+\left[\begin{array}{c}
\alpha_{x} \\
\frac{\alpha_{y} K_{x}}{x_{e q}}
\end{array}\right] \delta u,
$$

where $\delta x=x-x_{e q}, \delta y=y-y_{e q}$, and $\delta u=u-u_{0}$. Response of the output $\delta y$ to a step change in the input $\delta u$ can be obtained by considering the transfer function,

$$
\frac{\delta Y(s)}{\delta U(s)}=\frac{y_{e q}}{u_{0}} \frac{\gamma s}{(s+\gamma)^{2}} .
$$

In response to a step input, the output waveform is $\delta y(t)=$ $\left(y_{e q} / u_{0}\right) \gamma t \exp (-\gamma t)$. This response has a pulse-like shape with peak amplitude $y_{e q}\left(\right.$ as $u_{0}=1$ ), rise time $1 / \gamma$, and decay timescale $1 / \gamma$. As such, these analytical calculations confirm the trend reported in the above simulations (Fig. 2). 
Further, an implicit source of temperature robustness in the model is the supposition that both $x$ and $y$ are effectively degraded via dilution identically. If their effective degradation rates are dissimilar, say $\gamma_{x}$ and $\gamma_{y}$ for $x$ and $y$ respectively, then a possible difference in how they change with temperature may add temperature sensitivity in the properties.For example, the equilibrium value $y_{e q}=K_{x} \alpha_{y} \gamma_{x} /\left(\alpha_{x} \gamma_{y}\right)$ indicates that the temperature coefficient $Q_{10}$ lies in the range 0.3-3.4, which is an expanded version of the range estimated above. Therefore, the modeling assumption that $x$ and $y$ have the same degradation constant provides an inherent cancellation of temperature dependencies.

Finally, we studied the relation between temperature robustness and the perturbation classes for which the transient response can be invariant [4]. For Eqns. (4)-(5), it has been shown that the output response $y(t)$ to input $u(t)$ is invariant to the scale of the input $u \rightarrow p u$, where $p$ is a scalar. A temperature perturbation can perturb all reaction rate parameters. To gauge the extent to which the transient response can attenuate this, we used the invariance characterization method in [4] to search for parameters and their combinations for which this input-output response is invariant. A notable case of an invariance is when both production parameters are identically scaled $\left(\alpha_{x}, \alpha_{y}\right) \rightarrow\left(p \alpha_{x}, p \alpha_{y}\right)$, with remaining parameters unchanged. Basically, when $\left(\alpha_{x}, \alpha_{y}\right) \rightarrow$ $\left(p \alpha_{x}, p \alpha_{y}\right)$ in Eqns. (4)-(5), $(x, y) \rightarrow(x / p, y)$ recovers the same equations showing that input-output response is unchanged, assuming that perturbed system has equilibrated. Therefore, these parameters combine to promote temperature robustness of the transient response, reiterating their actions with respect to robustness of steady-state discussed above.

To summarize, this model already has three inherent features that can enhance the temperature robustness of the pulse height and the equilibrium value. These three are,

1) Similar temperature dependencies of production rates.

2) Proportionality to DNA binding constant, itself a ratio of two rates.

3) Same effective degradation terms acting on $x$ and $y$.

\section{DESIGNS FOR TEMPERATURE ROBUSTNESS}

With the aim of enhancing robustness of these properties to temperature, we explore modifications to this circuit. Motivated by the presence of negative feedback in numerous robustness contexts, we explored the effect of adding negative transcriptional feedback to the circuit.

1. Negative feedback of $y$ on itself. Consider a model, similar to above, where $y$ negatively feeds back onto itself,

$$
\begin{aligned}
& \frac{d x}{d t}=\alpha_{x} u-\gamma x, \\
& \frac{d y}{d t}=\alpha_{y} u \frac{K_{x}}{x} \frac{K_{y}}{y}-\gamma y .
\end{aligned}
$$

We find that the adaptation property can persist in this model (see Fig. 3A). In fact, the equilibrium value, $y_{e q}=$ $\sqrt{K_{x} K_{y} \alpha_{y} / \alpha_{x}}$. In comparing this value with the one obtained from the above model, we note the presence of a square root. This square root can be significant from the
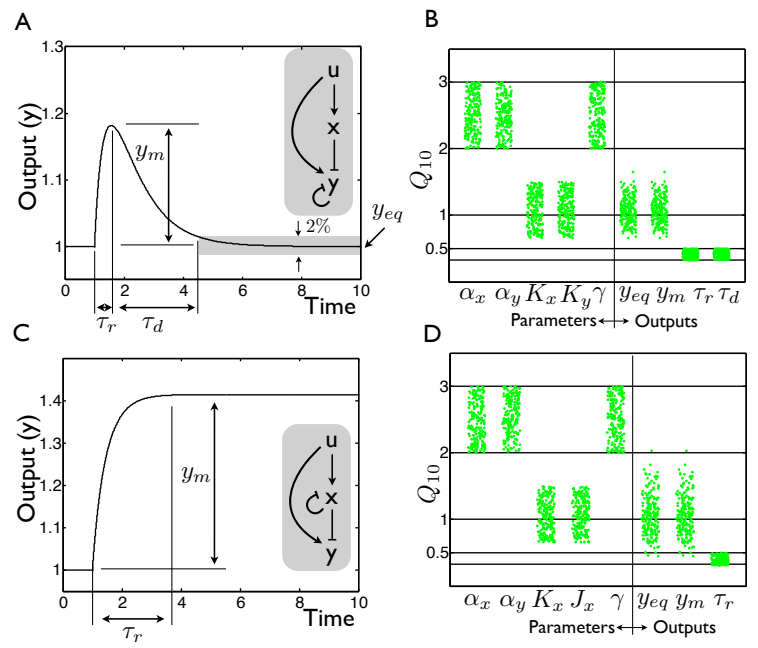

Fig. 3. Effect of different negative feedback configurations on the propagation of temperature dependence in an incoherent feedforward loop circuit. A-B. Negative feedback is added from $y$ onto itself. C-D. Negative feedback is added from $x$ onto itself. Simulations are performed as noted previously. Additional parameters $K_{y}=J_{x}=1$.

point of view of temperature robustness. If a rate $k$ has a $Q_{10}$ in the range $2-3$, then $\sqrt{k}$ has a $Q_{10}$ in the range 1.41-1.73, which indicates enhanced temperature robustness. Consistent with this, we find that the $Q_{10}$ of $y_{e q}$ should lie in the range $0.54-1.84$. This is a slightly narrower range than that obtained in the model without transcriptional feedback. Indeed, this is also seen when the $Q_{10}$ 's of parameters are chosen randomly (Fig. 3B). These computations show a slight enhancement in the temperature robustness properties of the adaptation value as well as the peak pulse amplitude. For these parameters, there is no significant difference in how the timescale properties of the pulse depend on temperature between this model and the main model. In general, the presence of the square root can have an effect of reducing the temperature dependence compared to the main model even if the production rates and binding constants have other temperature dependencies different from the ones assumed here. Likewise, negative feedback of $y$ on itself with higher co-operativity $\left(\left(K_{y} / y\right)^{n}, n>1\right.$ rather than just $\left.K_{y} / y\right)$ can further augment such a temperature robustness effect.

2. Negative feedback of $x$ on itself. Next, we consider the case where $x$ negatively feeds back on itself. The corresponding model is,

$$
\begin{aligned}
& \frac{d x}{d t}=\alpha_{x} u \frac{J_{x}}{x}-\gamma x, \\
& \frac{d y}{d t}=\alpha_{y} u \frac{K_{x}}{x}-\gamma y .
\end{aligned}
$$

Here, we find that the equilibrium value itself depends on the input, $y_{e q} \sim \sqrt{u}$. This dependence on the input indicates that the adaptation property itself does not exist. Nevertheless, we performed a similar analysis as above to gauge how temperature may affect output properties (Fig. $3 \mathrm{C}-\mathrm{D})$. We find that the output amplitudes corresponding to the maximum and final values, $y_{m}$ and $y_{e q}$, respectively, have 
a larger spread relative to temperature, whereas the timescale $\tau_{r}$ depends on temperature similar to the above cases.

These two models illustrate the effect of adding different negative feedback configurations with the aim of modifying temperature robustness of the pulse property of the incoherent feedforward loop circuit. Of the two, we find that the negative feedback from $y$ onto itself can help enhance temperature robustness of the amplitude properties.

Finally, we explored a case in which the temperature dependence of the circuit given by Eqn. (4)-(5) is known. For simplicity, we localize the temperature dependence to one parameter $\alpha_{x}$, assuming the remaining parameters to be independent of temperature. In this case, perfect temperature compensation can be achieved if a term such as $v=$ $-\left(\alpha_{x}(T)-\alpha_{x}\left(T_{0}\right)\right) u$ can be added to Eqn. (4). A possible biomolecular implementation of this is through the design of a promoter producing $x$, but with opposite temperature dependence.

\section{PRELIMINARY EXPERIMENTS}

As a step towards the experimental design of an incoherent feedforward loop circuit that is temperature-robust, we performed a preliminary experiment measuring the circuit dynamics of such a circuit inside cells at different temperatures. The goal of this experiment was to observe whether or not temperature affects the behavior of an actual circuit realization inside growing cells. This implementation of an incoherent feedforward loop uses transcriptional interactions (Fig. 4A): Transcription factor AraC can activate the expression of the transcriptional repressor TetR and the green fluorescent protein deGFP. Both proteins are expressed under the AraC-activable $P_{b a d}$ promoter. Additionally, TetR binding sites are inserted into the $P_{b a d}$ promoter controlling expression of deGFP so that it is repressible by TetR. An advantage of using the transcription factors AraC and TetR is that their activity can be modulated using inducers arabinose and anhydrotetracycline (aTc), respectively. Finally, AraC is expressed from a constitutive promoter that is regulated by the housekeeping sigma factor $\sigma^{70}$. We realized that this promoter was temperature-sensitive in that its expression increases with increasing temperature. Further, this sensitivity is a consequence of the activity of a mutant cI protein, which can additionally affect the growth rate. Nevertheless, we proceeded with measurements of this circuit to get a sense of whether and how temperature can affect the response.

Circuit dynamics were measured in a platereader (BioTek Synergy H1). A single colony from an LB plate was picked and grown overnight in a clear, rich media (MOPS-glycerol, Teknova M2105 with $50 \%$ glycerol instead of $20 \%$ glucose, final concentration $0.4 \%$ glycerol) at $29^{\circ} \mathrm{C}$. This liquid culture was diluted 1:50 into fresh media and grown for a second overnight cycle at the same temperature. Then, the culture was diluted 1:50 in fresh media and grown for around 2 hours at the same temperature. This culture was used for assays with appropriate amounts of inducers. Measurements were performed in a 96-well plate sealed with a BreatheEZ membrane. The plate reader protocol alternated double-

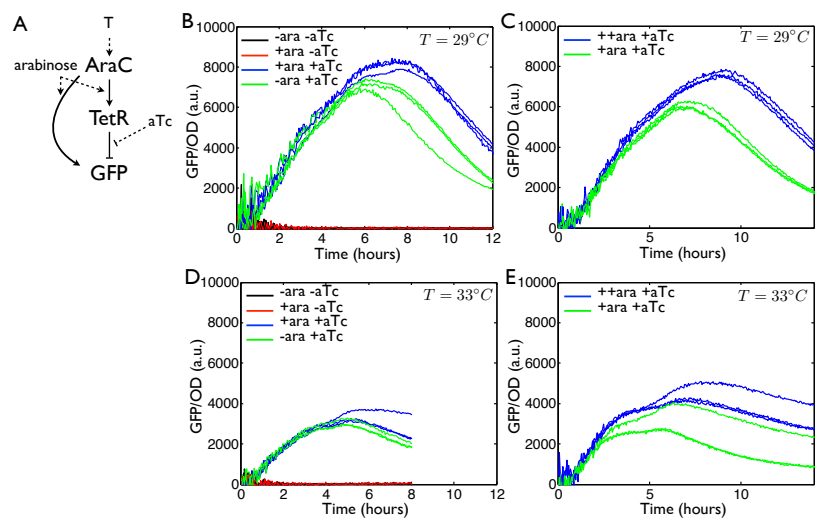

Fig. 4. Preliminary data characterizing the temperature dependence of an incoherent feedforward loop circuit. A. Schematic illustration of the circuit realization. B. and C. are measurements at $29^{\circ} \mathrm{C} \mathrm{D}$. and E. are measurements at $33^{\circ} \mathrm{C}$. In each panel, different colors represent different inducer combinations. ++ara denotes $0.018 \%$ arabinose. +ara denotes $0.0018 \%$ arabinose. $+\mathrm{aTc}$ denotes $0.001 \mu \mathrm{g} / \mathrm{ml}$ aTc. -ara and -aTc denote the absence of these inducers. Raw fluorescence and OD values were background subtracted using well with only media and no cells (autofluorescence was found to be similar to this blank fluorescence in this media). Each measurement was performed in triplicate on the same day.

orbital shaking with measurements at 3 minute intervals of the optical density (600 nm, denoted OD) and green fluorescent protein (excitation $485 \mathrm{~nm}$, emission $525 \mathrm{~nm}$ ) at $29^{\circ} \mathrm{C}$. All growth steps were performed with appropriate antibiotics.

We found that the circuit could exhibit a pulse response (Fig. 4B). Interestingly, this pulse response depended on the presence of the inducer aTc, with no pulse in the absence of aTc. This is likely due to the strong repressing effect of TetR. Additionally, there was a small increase in the height of the pulse as arabinose levels were increased, consistent with the expectation from the circuit diagram. To check this further, two different arabinose levels were used in the presence of aTc (Fig. 4C). As expected, pulse height increased with arabinose level. Overall, we found an expected pulse-like shape for this circuit, but the apparent dominant effect of aTc needed to be investigated further.

To investigate whether this response is temperature dependent, we repeated the above protocol at $33^{\circ} \mathrm{C}$ (Fig. 4D,E). The circuit exhibited a pulse-like shape at this temperature as well. However, we noted that the maximum pulse amplitude as well as the time taken to reach this amplitude were different. These results provide initial evidence for the dependence of both the pulse height and timescale of the response on temperature. We note the two non-idealities of this circuit realization due to the apparent dominant effect of the inducer aTc and the temperature-sensitive promoter expressing AraC. Indeed, addressing the latter through the use of a different promoter can directly reduce the effect of temperature on both promoter strengths and the growth rate, corresponding to the model parameters $\alpha_{x}, \alpha_{y}$ and $\gamma$, respectively. These measurements naturally raise questions of how to make this design more temperature-robust, by addressing these non-idealities as well as through the models 
analyzed in previous sections.

\section{CONCLUSIONS AND FUTURE WORK}

Design of biomolecular circuits whose function is robust to temperature is a key challenge. Here, we used control-theoretic principles to guide the goal of designing a temperature-robust pulse-generating incoherent feedforward loop circuit. First, we computationally studied a standard incoherent feedforward loop circuit using a structured uncertainty representation, pointing out inherent circuit features that promote robustness. Second, we investigated design additions that can improve this robustness, including negative transcriptional feedback at the output stage, and noted conditions where perfect temperature compensation can be possible. Third, we present preliminary experiments showing that the response of an initial incoherent feedforward loop circuit design can change with temperature. These results lay the groundwork for the design of a temperature-robust incoherent feedforward loop circuit.

An interesting aspect in achieving temperature robustness is how much knowledge of the temperature dependence of the circuit parameters is required. From one point of view, some mechanisms can promote temperature robustness without this information per se. An example is in the first design variant, where addition of a square root expression can reduce the $Q_{10}$ of the output. On the other hand, it is reasonable to expect knowledge of the temperature dependence to aid temperature compensation. As in examples of the production-degradation circuit and the feedforward loop circuit, this can cancel the effect of temperature through appropriate feedback. This seems similar to the Internal Model Principle, where an internal model of a disturbance in a system allows it complete disturbance attenuation.

A natural extension of this study is to proceed with the construction of feedforward loop circuit designs with different temperature-robustness properties. In addition to experiments in cells, the use of a cell extract-based transcriptiontranslation environment may also help by providing faster design cycles [21]. As part of this, a first step is to construct an alternative to the basic incoherent feedforward loop circuit to verify the predicted inherent temperature robustness properties. A second step is to add the negative transcriptional feedback at the output stage and check for the predicted enhancement in robustness. Additionally, it should motivate the development of mathematical tools for uncertainty representations as design aids.

Investigating the design of temperature-robust biomolecular circuits is part of a larger goal of designing biomolecular circuits that robustly function in a range of environments. We have presented foundational steps towards the design of a temperature-robust incoherent feedforward loop, a biomolecular circuit that is both widespread and exhibits rich dynamic behavior. These results should help in the design of other temperature-robust circuits as well as to further analyze temperature-robustness in naturally occurring biomolecular circuits.

\section{ACKNOWLEDGMENT}

We gratefully acknowledge S. Guo and C. Hayes for their help with the experimental part, especially for providing the $E$. coli strain containing the biomolecular circuit and the measurement protocol. We also thank the anonymous reviewers for their role in improving the manuscript.

\section{REFERENCES}

[1] N. Barkai and S. Leibler, "Robustness in simple biochemical networks," Nature, vol. 387, pp. 913-917, June 1997.

[2] S. Cardinale and A. P. Arkin, "Contextualizing context for synthetic biology-identifying causes of failure of synthetic biological systems," Biotechnol J, vol. 7, pp. 856-866, July 2012.

[3] M. Skataric and E. D. Sontag, "A characterization of scale invariant responses in enzymatic networks," PLoS Comput Biol, vol. 8, p. e1002748, Nov. 2012.

[4] O. Shoval, U. Alon, and E. Sontag, "Symmetry invariance for adapting biological systems," SIAM J Appl Dyn Syst, vol. 10, pp. 857-886, Jan. 2011.

[5] G. Shinar and M. Feinberg, "Design principles for robust biochemical reaction networks: What works, what cannot work, and what might almost work," Math Biosc, vol. 231, pp. 39-48, May 2011.

[6] R. Steuer, S. Waldherr, V. Sourjik, and M. Kollmann, "Robust signal processing in living cells," PLoS Comput Biol, vol. 7, p. e1002218, Nov. 2011.

[7] M. Nakajima, K. Imai, H. Ito, T. Nishiwaki, Y. Murayama, H. Iwasaki, T. Oyama, and T. Kondo, "Reconstitution of circadian oscillation of cyanobacterial KaiC phosphorylation in vitro," Science, vol. 308, no. 5720, pp. 414-5, 2005.

[8] J. Stricker, S. Cookson, M. R. Bennett, W. H. Mather, L. S. Tsimring, and J. Hasty, "A fast, robust and tunable synthetic gene oscillator," Nature, vol. 456, pp. 516-519, Nov. 2008.

[9] A. B. Reyes, J. S. Pendergast, and S. Yamazaki, "Mammalian peripheral circadian oscillators are temperature compensated," $J$ Biol Rhythms, vol. 23, pp. 95-98, 2008.

[10] O. Oleksiuk, V. Jakovljevic, N. Vladimirov, R. Carvalho, E. Paster, W. S. Ryu, Y. Meir, N. S. Wingreen, M. Kollmann, and V. Sourjik, "Thermal robustness of signaling in bacterial chemotaxis," Cell, vol. 145 , pp. 312-21, 2011.

[11] F. Hussain, C. Gupta, A. J. Hirning, W. Ott, K. S. Matthews, K. Josic, and M. R. Bennett, "Engineered temperature compensation in a synthetic genetic clock," Proc. Natl. Acad. Sci. U.S.A., vol. 111, pp. 972-977, Jan. 2014.

[12] S. Mangan and U. Alon, "Structure and function of the feed-forward loop network motif," Proc Natl Acad Sci USA, vol. 100, pp. 1198011985, Oct. 2003.

[13] S. S. Shen-Orr, R. Milo, S. Mangan, and U. Alon, "Network motifs in the transcriptional regulation network of escherichia coli," Nat Genet, vol. 31, pp. 64-68, May 2002.

[14] J. J. Tyson, K. C. Chen, and B. Novak, "Sniffers, buzzers, toggles and blinkers: dynamics of regulatory and signaling pathways in the cell," Curr Opin Cell Biol, vol. 15, pp. 221-231, Apr. 2003.

[15] W. Ma, A. Trusina, H. El-Samad, W. A. Lim, and C. Tang, "Defining network topologies that can achieve biochemical adaptation," Cell, vol. 138, pp. 760-773, Aug. 2009.

[16] L. Goentoro, O. Shoval, M. W. Kirschner, and U. Alon, "The incoherent feedforward loop can provide fold-change detection in gene regulation," Mol Cell, vol. 36, pp. 894-899, Dec. 2009.

[17] O. Shoval, L. Goentoro, Y. Hart, A. Mayo, E. Sontag, and U. Alon, "Fold-change detection and scalar symmetry of sensory input fields," Proc Natl Acad Sci USA, vol. 107, pp. 15995-16000, Sept. 2010.

[18] J. C. Doyle, B. A. Francis, and A. Tannenbaum, Feedback control theory. Mineola, N.Y.: Dover, 2009.

[19] E. D. Sontag, "Adaptation and regulation with signal detection implies internal model," Syst Control Lett, vol. 50, no. 2, pp. 119-126, 2003.

[20] T.-M. Yi, Y. Huang, M. I. Simon, and J. Doyle, "Robust perfect adaptation in bacterial chemotaxis through integral feedback control," Proc Natl Acad Sci USA, vol. 97, pp. 4649-4653, Apr. 2000.

[21] J. Shin and V. Noireaux, "An E. coli cell-free expression toolbox: application to synthetic gene circuits and artificial cells," ACS Synth Biol, vol. 1, pp. 29-41, Jan. 2012. 